\title{
Lab-based radiometric concepts for undergraduate and graduate students
}

\section{Emmett lentilucci}

Emmett J. lentilucci, "Lab-based radiometric concepts for undergraduate and graduate students," Proc. SPIE 11143, Fifteenth Conference on Education and Training in Optics and Photonics: ETOP 2019, 111432E (2 July 2019); doi: $10.1117 / 12.2523868$ 


\title{
Lab-based Radiometric Concepts for Undergraduate and Graduate Students
}

\author{
Emmett J. Ientilucci \\ Rochester Institute of Technology, Chester F. Carlson Center for Imaging Science \\ Rochester, NY 14623 USA
}

\begin{abstract}
Radiometry is formally defined as the science of characterizing or measuring how much electromagnetic energy is present at, or associated with, some location or direction in space. It involves the measurement and quantification of optical flux. It has evolved separately in the fields of physics, illumination or vision, and engineering, and as a result a host of terms are used to describe various radiometric concepts. Over the years there have been various texts on the subject to help demystify some of these concepts with less emphasis on "practical" radiometry (i.e., meaning laboratory examples). At the Rochester Institute of Technology (RIT), an entire course has been devoted to the subject along with a dozen or so hands-on laboratory exercises. This paper will discuss our approach to the dissemination of "practical" radiometry through exercises that talk about construction of radiometers, inverse square law, radiometric and spectral calibration (of a spectrometer), diffraction gratings, blackbodies and sources, spectral transmission and reflectance measurements, detector spectral responsivity measurements, transfer calibration, and more.
\end{abstract}

Keywords: Radiometry, Calibration, Spectral Calibration, Inverse Square Law, Transfer Calibration, Optical Radiation Measurements, Undergraduate Lab, Graduate Lab

\section{INTRODUCTION}

The Chester F. Carson Center for Imaging Science (CIS) is a research and education Center with in the College of Science (COS) at the Rochester Institute of Technology (RIT) which offers BS, MS and PhD degrees in Imaging Science. The program is very interdisciplinary and involves study and research in all aspects of imaging. From how light is generated to how the world is perceived, Imaging Science addresses questions about every aspect of systems that are used to create, perceive, analyze, and optimize images. ${ }^{1}$ This includes domain areas such as remote sensing and satellite systems, human vision perception, optics, computer vision, astronomy and space science, nanoimaging and materials, detector research and imaging of cultural artifacts.

A major, as interdisciplinary as this one, requires students with proficient skills in math, physics, and computer programming. A sampling of courses taught (in no order) include those heavy in imaging systems, vision, color science, linear systems, optics, interaction with light and matter, computer vision, detectors, noise, image processing, radiative transfer, and radiometry. In particular, radiometry is considered one of the "core" courses in the program and is taught very early in the undergraduate and graduate curriculums. This course is paramount in Imaging Science since it teaches all the important, necessary, vernacular used in subsequent courses.

Thus, this paper will discuss how we approach the dissemination of information related to the field of radiometry to our Imaging Science students, regardless of their area of research. In particular, we will turn our attention to the hands-on radiometry labs we have designed over the years.

Further author information: Emmett J. Ientilucci

E-mail: Emmett@cis.rit.edu, Telephone: (585) 475-7778

Fifteenth Conference on Education and Training in Optics and Photonics: ETOP 2019, edited by

Anne-Sophie Poulin-Girard, Joseph A. Shaw, Proc. of SPIE Vol. 11143, 111432E · (C) 2019

SPIE, ICO, IEEE, OSA · CCC code: 0277-786X/19/\$18 · doi: 10.1117/12.2523868

Proc. of SPIE Vol. 11143 111432E-1 


\section{RADIOMETRY AT THE CENTER FOR IMAGING SCIENCE}

\subsection{The Radiometry Lecture}

As previously mentioned, the core course, radiometry, is taught early on in both the undergraduate and graduate curriculums. We really need the students to understand the concepts involved in characterizing or measuring how much electromagnetic energy is present at, or associated with, some location or direction in space. The undergraduate and graduate versions of the course do differ slightly. At the undergraduate level, we tend to cover the material at a slower pace and, as a result, cannot cover as much material in the same 15-week semester. The subject matter currently comes from a developing text book on the subject ${ }^{2}$ where we cover topics such as:

- Optical Radiation and the Nature of Light

- Radiometric Quantities and Units (i.e., point sources, solid angle, projected area, energy flux, irradiance exitance, intensity, radiance, intro to photometry, and general spectral considerations)

- Blackbody Radiation (Planck's Law, approximations, Wien Law, Stefan-Boltzmann Law, numerical integration and the Planck function, non-blackbodies, emissivity, Kirchhoff's Law, types of blackbodies (plate, cavity, etc.) and introduction to the concept of calibration)

- Sources of Radiation (gas discharge and physics of operation, color rendering, luminous efficacy, fluorescent sources, line broadening, incandescent sources, thermionic emission, lasers, and LED's)

- Irradiance Variation with Distance and Angle (all the variation of the inverse square law and falloff from point- line- and extended area sources)

- Radiance and Lambertian Surfaces (radiance invariance, responsivity, Lamberts Cosine Law, relationships between radiance and exitance as well as flux and intensity)

- Detectors (thermal, photo-emissive, and photon detectors, operation and usage)

- Photometry and Color Temperature (human visual system, terminology, color matching function, CIE curve, Planckian locus, color temperature)

- Detector Figures of Merit (responsivity, noise, SNR, NEP, D, D-star, spectral response, 2D arrays, and QE)

- Spectroradiometry (spectral considerations, prisms, filters, diffraction gratings and instrumentation)

- Properties of Materials (general material properties, BRDF, and polarization)

- Radiometry and Imaging Systems (lens falloff, irradiance on the focal plane, and the camera equation)

- Radiometric System Calculations (sensor performance parameters, shot noise, detector-sensor performance calculations for the visible and thermal regions)

- Integrating Spheres (ideal integrating spheres, real integrating spheres, total flux, laser power measurements, collection of transmisttance, reflectance, and uniform sources for calibration)

- Area or Non-Point Sources (definition of area or extended source, energy exchange between parallel disc's, parallel planes, and arbitrary surfaces, camera equation for any f-number, radiation transfer from the sun)

- Radiometry in Turbid Media (radiometry related to atmospheric absorption, self-emission, and scattering)

- Radiation Propagation Models (propagation models and radiative transfer (RT) codes, optical properties of the atmosphere, single and multiple scattering)

This material is currently distributed to students as a 400-page set of notes (i.e., a working manuscript ${ }^{2}$ ). Each topic listed above includes example problems (in the notes) and homework problems at the end of each chapter. 

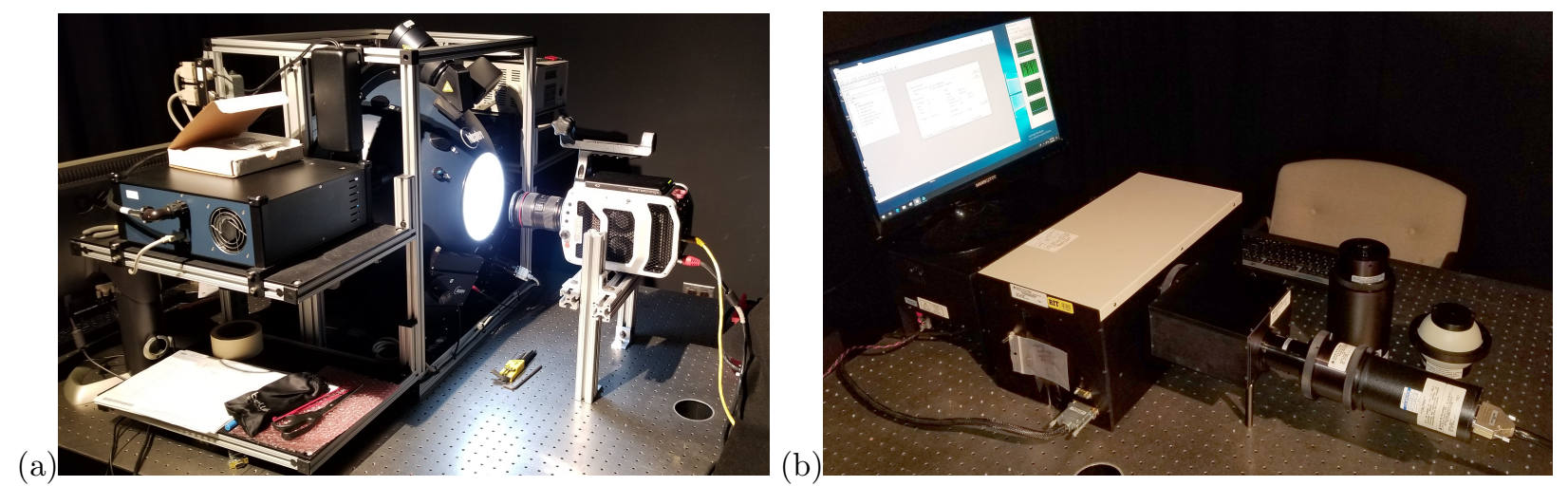

Figure 1. (a) NIST-traceable integrating sphere and (b) Optronics Labs OL-750 Monochromator which resides in the Optical Calibration Facility in the Chester F. Carlson Center for Imaging Science.

\subsection{The Optical Calibration Facility (OCF) at RIT}

Coupled with the lecture topics listed above, which are under development, we give the students an opportunity to work in a laboratory setting. The Imaging Science Center currently houses a variety of small optics labs for students to work in as well as our new Optical Calibration Facility (OCF). The new calibration facility is a strategic asset for both research and academics in the Center. We have acquired a NIST-traceable, custom 20inch integrating sphere from Labsphere, Inc., as seen in Figure 1(a). This is the heart of our current radiometric calibration capability in terms of flat fielding, linearity assessment and absolute calibration. In addition, we recently acquired an Optronics Labs OL-750 Automated Spectroradiometric Measurement System, shown in Figure 1(b). This allows us to perform spectral measurements, such as relative spectral response (RSR) from 0.4 to 2.5 microns on camera and single-element detector (i.e., photodiodes) systems. We also purchased three 1000 watt FEL lamps (along with FEL lamp holders) to be used in a lamp-plaque or lamp-detector calibration configuration. Only one of the FEL lamps is calibrated and is NIST-traceable with an uncertainty less than $1 \%$ in the visible. We will transfer the calibration to the other two "working standards" as soon as the precision current supply arrives (i.e., June 1, 2019). Lastly, we have the capability to assess wavelength calibration using our Argon and Mercury (Neon) set of spectral emission lamps. Future outlook for the lab and curriculum integration includes the incorporation of a collimator (VIS-LWIR) so as to perform spatial detector/imaging system analysis and calibration along with the development of a course on radiometric measurements and calibration.

\subsection{The Radiometry Lab Experience}

The previous two sections touched upon what is taught in our radiometry lecture along with descriptions of instrumentation in our state-of-the art calibration facility. In this section, we discuss the actual student lab experience and the type of laboratory exercises given related to radiometry.

We have currently assembled nine radiometry labs with another two under development. These labs are currently assembled in a 100-page laboratory manual handed to students at the start of the semester. In this section, we will show the list of laboratory exercises followed by brief explanations of each one. The current list of radiometry laboratory exercises is as follows:

1. Overview: Semi-Conductor Physics and Using a Silicon Photodiode

2. Building a Radiometer

3. Using your Radiometer

4. Verification of the Inverse Square Law with your Radiometer

5. Detector Broadband Radiometric Calibration with your Radiometer

6. Concept of Diffraction Gratings 
7. Spectrometer Spectral Calibration

8. Spectrometer Radiometric Calibration and Determining the Distribution Temperature of an unknown Source

9. Making Spectral Measurements: Sources, Transmission, and Reflection

10. (Under development) Determine the Relative Spectral Response (RSR) of a Photodiode

11. (Under development) Source Based Calibration using and FEL Lamp

Before we go into detail about the laboratory exercises, we note that there is an expectation that students model their lab reports similar to that of a single column SPIE proceeding or a two column IEEE article. They can use any word processing software (i.e., MS Word), though we encourage the use of LaTeX. Lastly, we note that at the end of each laboratory handout exists a rubric used to guide students (and graders) on expected outcomes and grading of the lab. An example rubric can be seen in Figure 2.

In the sections below, we introduce the current set of labs related to our radiometry course.

\subsubsection{Overview: Semi-Conductor Physics and Using a Silicon Photodiode}

This first lab is used to get the students familiar with the key component of their radiometers (to be constructed in the next lab), the silicon photodiode. The lab is basically a handout the students read, followed by a series of questions. In the lab we first discuss some background information about semi-conductor physics followed by the construction of a silicon photodiode. We illustrate how the photodiode can be used in a broad-band radiometer. The semi-conductor background discusses how we dope silicon to create n- and p-type semiconductors, at least to the level pertinent in the class. Of course, an entire course can be devoted to this subject matter so we only touch up what is essential. We then talk about how we put these semiconductors together to create a pn-junction. We note that the simplest device that incorporates a pn-junction is called a diode and that a light sensitive variant of the diode is called a photodiode. This is followed by discussion on how we can amplify the output of the photodiode using an operational amplifier. Lastly, we briefly introduce the concepts of photodiode spectral response, responsivity and quantum efficiency. The lab handout ends with a series of 14 questions to be answered and handed in.

\subsubsection{Building a Radiometer}

Once we have exposed the students to the basic workings of photodiodes and how they can be used to sense light, we actually have them construct a working radiometer. The radiometer utilizes a UDT silicon photodiode with a BNC connection, an LM741 operational amplifier, two 9-volt batteries, a series of feedback resisters, a digital multi-meter, and a 5-position DIP switch. The entire assembly is fabricated on a breadboard using the circuit design shown in Figure 3(a). The operational amplifier is powered by a bi-polar supply, though this is not completely necessary, and is connected to the photodiodes cathode terminal. The resister network allows for a large range of gains when operating the device. That is, low gains for high light levels and high gains for low light levels. The output voltage is read directly from a multi-meter with a saturation value around 9-volts (i.e., the power supply voltage). Currently, the UDT photodiode does not have a diffuser attachment associated with it (i.e., for cosine correction behavior and hemispherical irradiance measurements). We simply expose the bare $1 \mathrm{~cm}^{2}$ active surface to illumination. This, along with hardware to form a solid angle for radiance measurements, will show up in our next round of improvements.

Before students come to lab, we encourage them to get familiar with the circuit before hand by exploring AutoDesk's TinkerCAD online. This website allows students to assemble and play with the circuit in a virtual world and has been found to be quite useful. 


\section{COURSE: Radiometry}

Due Date:

Name:

Lab Partners:

Lab \# and Name: _ Detector Broadband Radiometric Calibration

\section{CONTENTS}

Introduction and/or Abstract and Conclusions

LUT for 4 resistors

Calibration curves for 4 resistors

Mean Gains

\section{DISCUSSION}

Answer questions in lab handout plus additional discussion (there are 7 questions listed in lab)

\begin{tabular}{ll}
$\begin{array}{ll}\text { Total Possible } \\
10.0\end{array}$ & TOTAL \\
\hline 15.0 & \\
\hline 18.0 & \\
\hline 7.0 & \\
\hline
\end{tabular}

40.0

\section{GENERAL FORMAT}

Format (includes extraneous data, syntax, layout, spelling)

Clarity / understanding of material

LATE

Extensively LATE (more than 2 weeks)

\section{0}

$\frac{-10.0}{-20.0}$

$-20.0$

Total: 100

Undergraduate Laboratory Manual

Page 60

Dr. Emmett J. Ientilucci 2018 @

Figure 2. Example rubric used to illustrate outcomes and grading of the lab. 


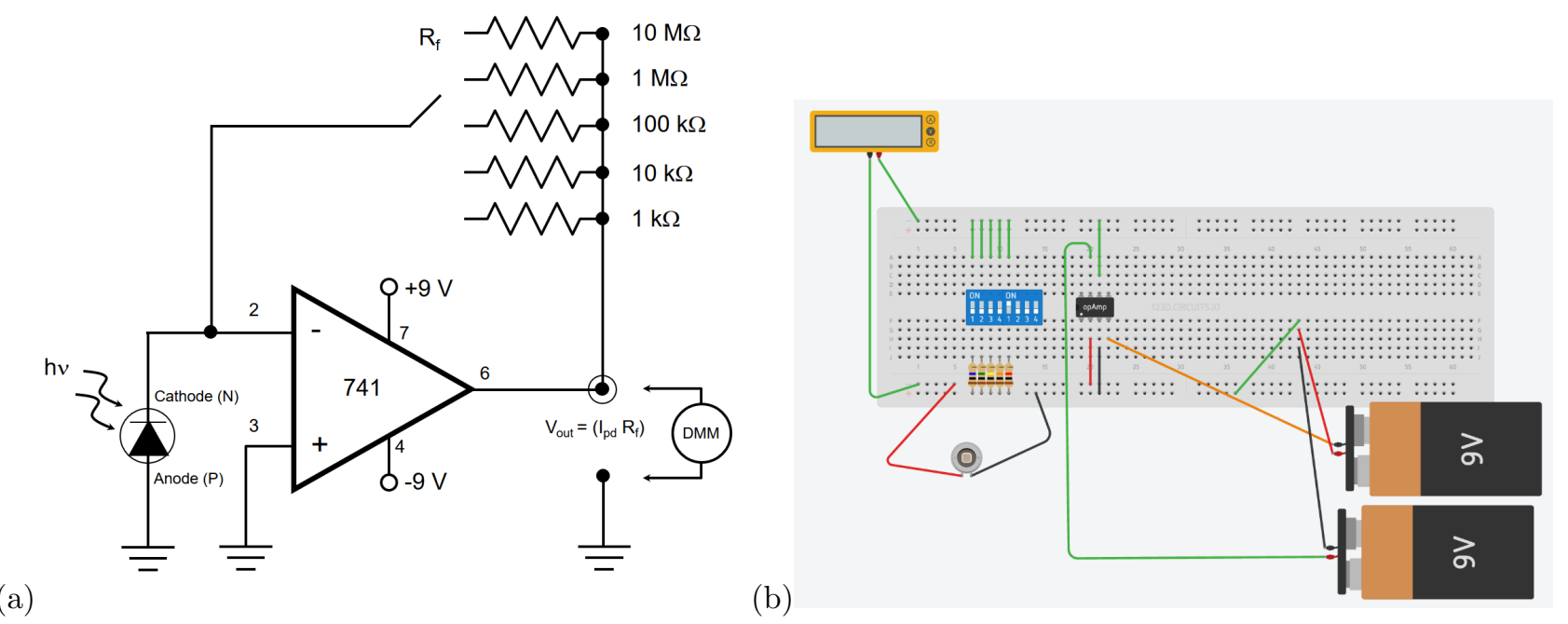

Figure 3. Radiometer (a) circuit and (b) breadboard layout.

\subsubsection{Using your Radiometer}

Once the radiometer has been constructed and tested for operation (i.e., it responds to light appropriately), we ask the students to quantify its behavior. This is accomplished by examining the radiometers gain and linearity as a function of illumination. We also examine dark current noise. For gain, there is a fixed light level incident on the radiometer followed by recording the output voltage, for each feedback resister setting. The voltage should change by orders of 10 , since this is how the feedback resister values were selected (i.e., differing by orders of 10). Given the measured resistance and known voltage, they can also calculate the current to see how this changes or doesn't change. Linearity is examined by reducing the light level in a predictable manor and examining the voltage output. Since the source is not calibrated, we set the source to a particular level and reduce the light onto the detector through use of known neutral density (ND) filters, since the relationship between ND and transmission is well known as $N D=-\log (\tau)$. In the end, the student can create a plot of voltage out of the detector versus ND (though it is really $\log$ ND to make it linear on the x-axis). From this, students can see if the changes in light level correspond to linear steps in output voltage.

\subsubsection{Verification of the Inverse Square Law (ISL) with your Radiometer}

Without a doubt, one of the cornerstone principles in radiometry is the falloff in radiation as a function of distance. The falloff experiment is fairly straight forward in that students simulate a bright point source and take voltage reading as a function of distance. There are some additional variables that are explored like non-point source behavior and using LED's as a source. Students plot the actual measured voltage from the un-calibrated radiometer, the predicted voltage (using the ISL), and a curve fit with a power model to see how close the exponent is to 2.0 (see Figure 4).

\subsubsection{Detector Broadband Radiometric Calibration with your Radiometer}

Up to this point, none of the previous labs required the student constructed radiometers to be calibrated. In this lab we illustrate the concept of generating a radiometric calibration look-up-table (LUT) that maps voltage to known irradiance, for all the radiometer feedback resistor gain settings. For this lab we do not have a calibrated light source, however, we do own a calibrated International Light Technologies (ILT) radiometer that measures irradiance (with the cosine corrector), thus we measure the irradiance of the (point) source at a distance of say, $30 \mathrm{~cm}$. We position the student constructed radiometer at this location (as well as a few other distances, say, 60, 90 , and $120 \mathrm{~cm}$ ) and capture voltage readings (as a function of distance). As mentioned in lab 2, we currently do not employ a cosine corrector on the device. This will appear in our next round of improvements. None-the-less, we can still effectively convey concepts related to calibration.

We use the neutral density (ND) filters to help generate a large number of data points (since we know the predicted transmissions of the filters, see the "Using your Radiometer" lab). Finally, we use the inverse square law 


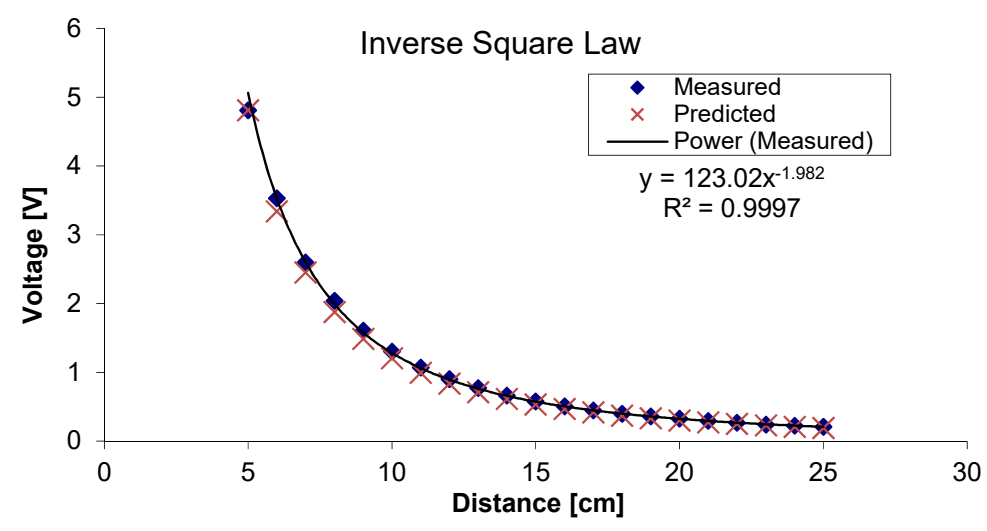

Figure 4. Results from inverse square law experiment using a student constructed radiometer.

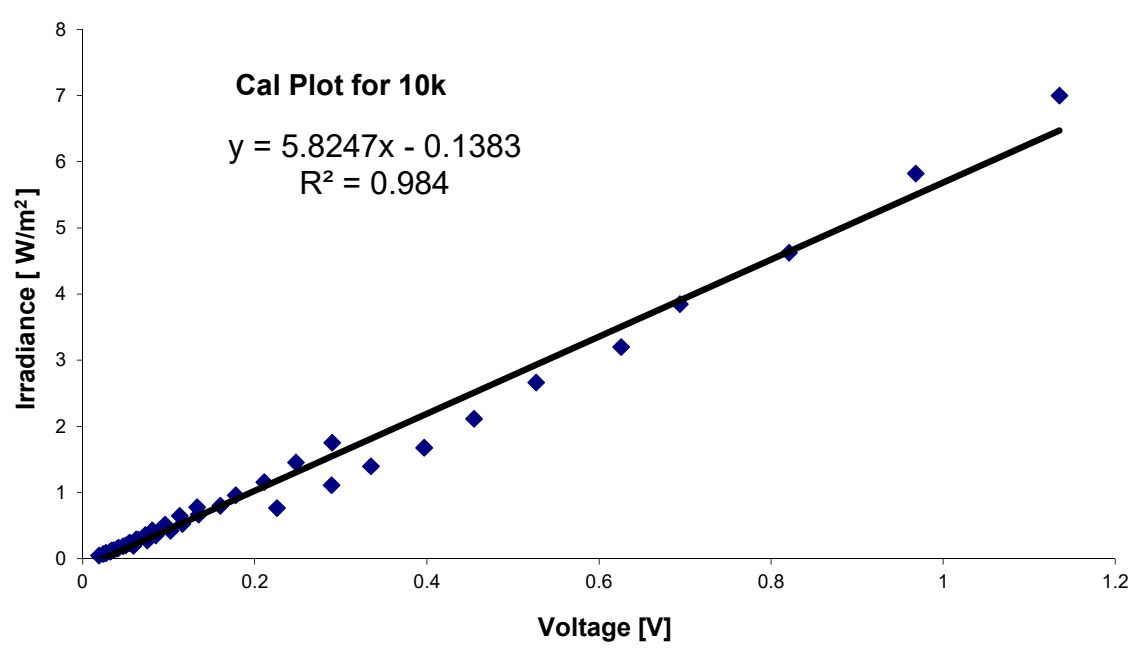

Figure 5. Calibrating the student-constructed radiometer. LUT mapping of radiometer voltage to irradiance.

(since it was so accurate in the previous lab) to predict the irradiance-like behavior at the various distance/ND pairs. The end result is a LUT (and calibration equation) that maps voltage to irradiance, as shown in Figure 5 for the 10k ohm feedback resistor gain setting.

\subsubsection{Concept of Diffraction Gratings}

The goal of this lab is to introduce the concept of diffractions gratings and spectral radiometry, which is also the subject of labs 7 and 8 . We introduce the grating equation, $m \lambda=d\left(\sin \theta_{i} \pm \sin \theta_{r}\right)$ and use it in both transmission and reflection grating set ups. For the transmission set up, we estimate the wavelength of a laser. Once this is known, the same laser is used to determine the groove spacing between tracks in a compact disc (CD) followed by a DVD. Basically, we are demonstrating how these ruled surfaces act like reflective diffraction gratings. The set up for the laser and track spacing determination can be seen in Figure 6. Turns out, this experiment provides fairly accurate results for the track spacing. In one experiment, the CD track spacing was found to be $1.65 \mu \mathrm{m}$ (or $606 \mathrm{LPM}$ ), an error of $3.1 \%$ from the industry standard spacing of $1.6 \mu \mathrm{m}$. For the DVD, example results were shown to be $712 \mathrm{~nm}(1404 \mathrm{LPM})$ which resulted in an error around $3.7 \%$ from the standard spacing of 740 $\mathrm{nm}$. 


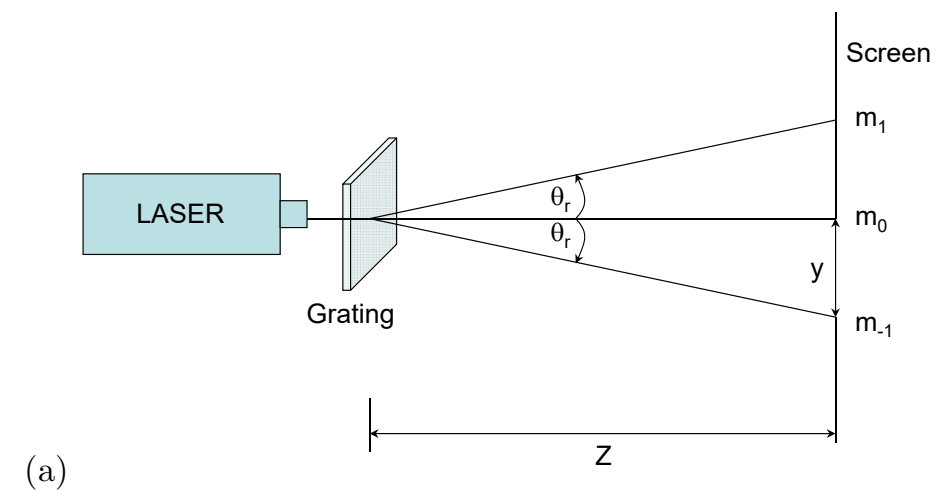

(b)

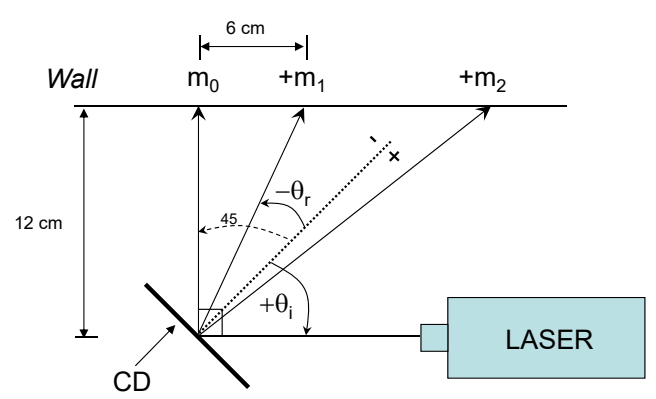

Figure 6. Diffraction grating set ups for determining (a) laser wavelength and (b) CD/DVD track spacing.

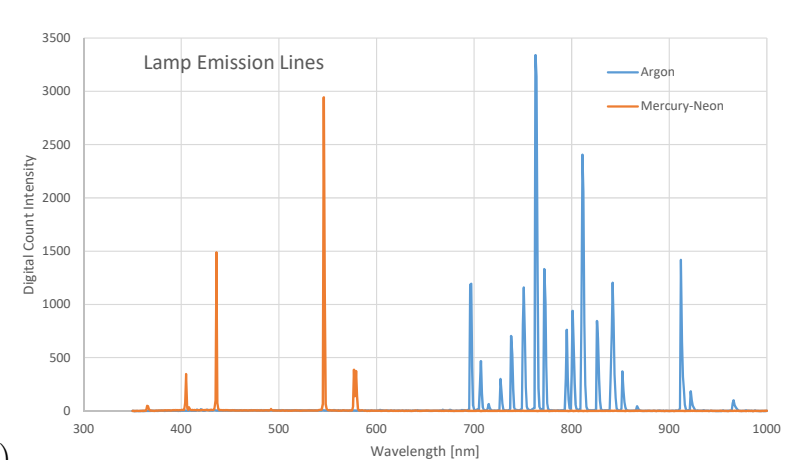

(a)

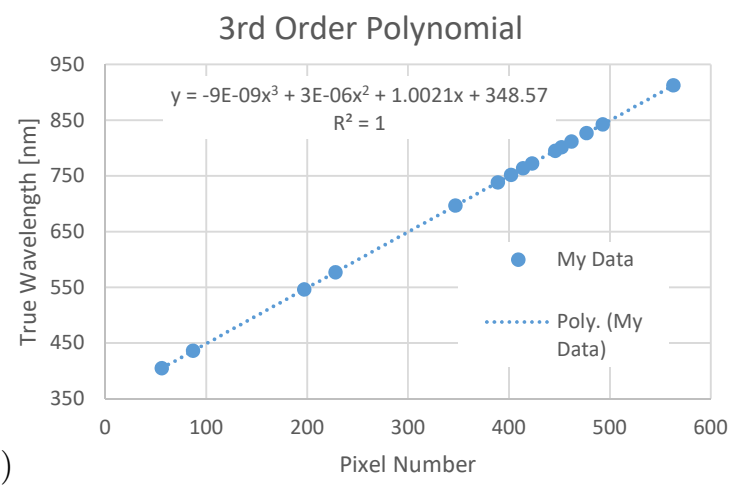

Figure 7. (a) Emission lines from Argon and Mercury-Neon sources and (b) 3rd order polynomial LUT mapping pixel to wavelength.

\subsubsection{Spectrometer Spectral Calibration}

This lab addresses the concept of spectral wavelength calibration of a point spectrometer. Students learn how to spectrally calibrate their Ocean Optics USB650 (of which we have five) using specialized, commercially available, light sources that emit sharp spectral lines at very well known wavelengths (which are published in the literature). The sources are measured with the USB650 and students eventually determine the relationship between pixel number (i.e., detector element) and wavelength using an $n$-degree polynomial. This is captured as a look-uptable (LUT) mapping pixel index to wavelength. The sources we use for this lab are Argon and Mercury-Neon which provide sharp emission lines across the full region from 300 to $1000 \mathrm{~nm}$, as seen in Figure 7(a). The polynomial we end up using is of degree three and takes on the form $\lambda(p)=\alpha_{1} p+\alpha_{2} p^{2}+\alpha_{3} p^{3}+b$ where we have wavelength, $\lambda$, as a function of pixel number, $p$. The coefficients, $\alpha_{1}, \alpha_{2}, \alpha_{3}$ along with intercept, $b$, need to be determined based on a programmed regression routine. The intercept, $b$, in our wavelength vs. pixel index LUT, would be the wavelength of pixel one, for example. The coefficients have units of $\mathrm{nm} / \mathrm{pixel}, \mathrm{nm} / \mathrm{pixel}^{2}$ and $n m / p_{i x e l^{3}}$, respectively.

\subsubsection{Spectrometer Radiometric Calibration and Determining the Distribution Temperature of an unknown Source}

Concepts learned in this lab involve utilization of a hand-held spectrometer, radiometrically calibrating the spectrometer, programming the Planck function and estimating the distribution temperature of an unknown incandescent source. Students first use the Ocean Optics USB650 to measure the (un-calibrated) spectrum (in digital counts) of a tungsten light bulb, often reflecting off a 100\% reflecting Lambertian Spectralon plaque. They then use a known calibrated radiance source to calibrate or "transfer the calibration" to the spectrometer, as illustrated in Figure 8. 


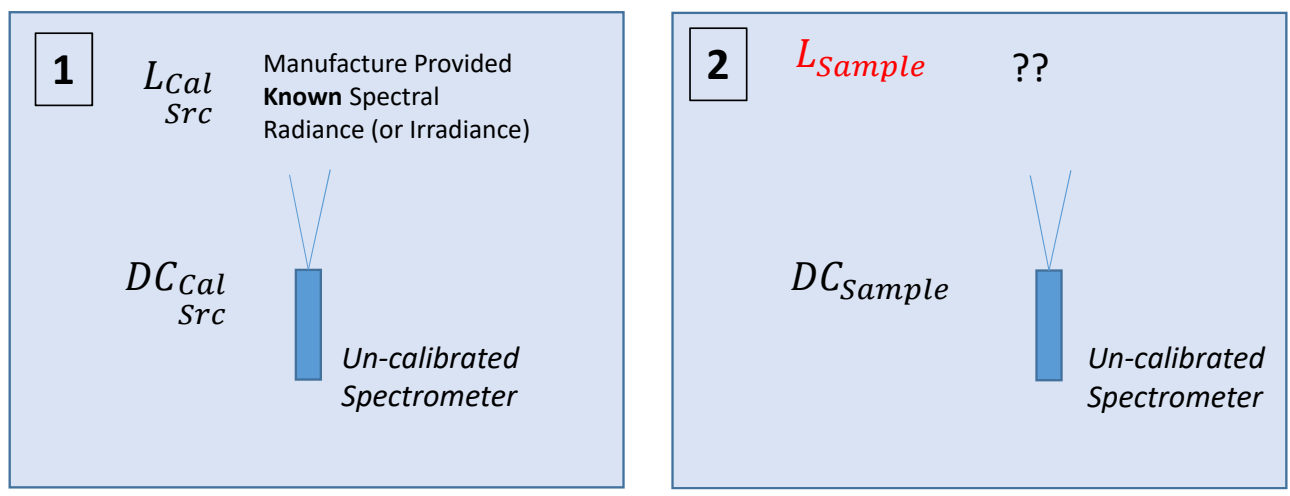

Figure 8. Transfer calibration. The un-calibrated (but stable from measurement-to-measurement) spectrometer is first used to measure a known radiance output in digital counts. This is followed by measuring the unknown sample, in digital counts, in which the spectral radiance can be determined.

Given the measurement scenario in Figure 8, we have three known quantities and one unknown quantity (i.e., the radiance of the sample in question). This can be expressed as

$$
\frac{L_{\text {calsrc }}}{D C_{\text {calsrc }}}=\frac{L_{\text {sample }}}{D C_{\text {sample }}}
$$

To be more complete and accurate, we should subtract off the noise measurement, thus we have

$$
\frac{L_{\text {calsrc }}}{D C_{\text {calsrc }}-D C_{\text {noise }}}=\frac{L_{\text {sample }}}{D C_{\text {sample }}-D C_{\text {noise }}}
$$

We can then solve for the calibrated absolute spectral radiance of the sample as

$$
L_{\text {sample }}=\frac{L_{\text {calsrc }}}{D C_{\text {calsrc }}-D C_{\text {noise }}} D C_{\text {sample }}-D C_{\text {noise }} \quad\left[\frac{\mu W}{\mathrm{~cm}^{2} n m}\right]
$$

This is essentially transferring the calibration from the known source to the USB650 and is often called transfer calibration. What is happening here is, we are using the ratio $L_{\text {calsrc }} / D C_{\text {calsrc }}$ to appropriately scale our initial, un-calibrated, digital count measurement of our source $\left(D C_{\text {sample }}\right)$ so as to produce a calibrated measurement of our sample $\left(L_{\text {sample }}\right)$.

Following this calibration, students measure the spectral distribution of an unknown tungsten source. They then program (MatLAB, Python, etc.) the Planck function, normalize the source measurement and Planck function data, followed by iterative curve fitting by varying the Planck temperature until and RMS error is minimized. Once minimized, it is fairly straight forward to determine what the distribution temperature value was for the unknown source, which should be around $2800-3000 \mathrm{~K}$.

\subsubsection{Making Spectral Measurements: Sources, Transmission, and Reflection}

This lab gives students hands-on experience using spectrometers to make spectral measurements of light sources, as well as measurements of transmissive and reflective materials. To start, we repeat the process of radiometrically calibrating the spectrometer, as was illustrated in the previous lab. Once calibrated, students then measure a variety of light sources (though they are not told what they are measuring) including a black light, blue LED, CFL, fiber optic light source, green LED, red LED, room/ceiling lights, standard tungsten bulb, and a white LED. Questions are posed about what types of sources they are examining in addition to the physics of operation (i.e., how do these sources generate light). 
The spectrometer is then used to perform transmission measurements of a variety of filters. These include ND filters, cut-off filters, cut-on filters, and the V-lambda filter (i.e., the human visual response). The filter descriptions are not provided to the students ahead of time. The students first measure the stable light output from a small integrating sphere with a 1-inch port. The second measurement is of the integrating sphere port but with the filter in the light path. It is explained that the ratio of these two measurements provides us with an estimate of the spectral transmission. Furthermore, is explained that the spectrometer need not be absolutely radiometrically calibrated for such measurements since this is ratio. Lastly, sources of error, including BRDF effects, are explained.

The last station is set up to perform (rough) spectral reflectance measurements. We say rough because proper total reflectance measurement requires the use of a certain integrating sphere set up, which we do not have for this experiment. In lecture, we would have already discussed issues related to specularity and the use of a goniometer to measure the reflectance at every exiting angle in the hemisphere above the sample, which is in contrast to what we are doing in this exercise. In this lab, we approximate hemispherical reflectance measurements by using a 45/0 measuring geometry. That is, we illuminate the sample at 45 degrees while measuring the signal from the sample at zero degrees. In this way, we avoid measuring any specular highlights. Here, the reflectance is computed as the ratio of two measurements: one of the illuminated sample and one from an illuminated $100 \%$ reflecting Lambertian Spectralon surface (i.e., a measurement of the source only). We discuss the pitfalls of performing these measurements outdoors where the source (i.e., the sun) is constantly changing and may not be exactly the same in both measurements and the need for the spectrometer to be stable but not necessarily calibrated in an absolute radiometric sense, again, since this is a ratio measurement.

\subsection{Radiometry Labs Currently Under Development}

In this section we briefly touch upon some of the newer radiometric labs we have been working on.

\subsubsection{Determine the Relative Spectral Response (RSR) of a Photodiode}

At the core of the student-built radiometers is the UDT silicon photodiode. The essence of this lab would be to have students actually measure the spectral response of the detector using the OL-750 monochromator. Armed with this response information, they can perform band-effective calculations such as determining the band-effective sensor radiance/iradiance given a spectral radiance/irradiance distribution, for example. This can then be compared to what the calibrated detector (i.e., photodiode) is actually reading.

\subsubsection{Source Based Calibration using and FEL Lamp}

This lab will teach students about calibration using a calibrated source. Namely, our 1000 watt calibrated FEL lamps in combination with a Spectralon plaque. This calibration set up is an alternative to using an expensive integrating sphere for radiometric calibration but does have some caveats. The idea is to give students the experience in setting up an FEL lamp (lab set up, alignment, use of special jig, operation of power supply, etc.) at the given calibrated distance $(i . e ., 50 \mathrm{~cm})$, to a Spectralon plaque so as to generate a source of spectral radiance. This source of radiance can then be used to calibrate detectors or imaging systems. Results can be cross-compared to using the integrating sphere as a means of stable spectral radiance, for example.

\section{SUMMARY REMARKS}

In this paper, we discussed why radiometry is such an important subject in the field of image science at the Chester F. Carlson Center for Imaging Science. We then described the current radiometry course outline, both from the lecture and lab point of view with heavy emphasis on the latter. By no means can we condense a 100-page laboratory manual down to a handful of pages for this particular publication. Instead, the hope was to give some insight as to how we teach lab-based radiometric concepts to students studying Imaging Science.

Though the current set of labs is somewhat comprehensive, we are still refining exercises generating at least one new lab (or a revamp of an existing lab) per year. Recently the focus has been on disseminating the concepts of detector-based and source-based calibration to students. This continues to evolve as we acquire more relevant equipment in our new Optical Calibration Facility (OCF). 


\section{ACKNOWLEDGMENTS}

The author would like to personally thank Professor Joseph Shaw for informing me about the ETOP 2019 meeting and inviting me to speak at the conference.

\section{REFERENCES}

[1] RIT-CIS, "Imaging Science Overview." http://www.cis.rit.edu/about/overview. (Accessed: May 2019).

[2] Ientilucci, E. J. and Schott, J. R., [Radiometry and Radiation Propagation], Oxford University Press, Oxford, UK (To be published).

Proc. of SPIE Vol. 11143 111432E-11

Downloaded From: https://www.spiedigitallibrary.org/conference-proceedings-of-spie on 26 Apr 2023 Terms of Use: https://www.spiedigitallibrary.org/terms-of-use 